\title{
Pathobiological Subtypes of Alzheimer Disease
}

\author{
Kurt A. Jellinger \\ Institute of Clinical Neurobiology, Vienna, Austria
}

\section{Keywords}

Alzheimer disease - Subtyping - Regional vulnerability .

Tau pathology · Alzheimer variants · Co-pathologies .

Key network destructions

\begin{abstract}
Alzheimer disease (AD), the most common form of dementia, is a heterogenous disorder with various pathobiological subtypes. In addition to the 4 major subtypes based on the distribution of tau pathology and brain atrophy (typical, limbic predominant, hippocampal sparing, and minimal atrophy $[M A]$ ), several other clinical variants showing distinct regional patterns of tau burden have been identified: nonamnestic, corticobasal syndromal, primary progressive aphasia, posterior cortical atrophy, behavioral/dysexecutive, and mild dementia variants. Among the subtypes, differences were found in age at onset, sex distribution, cognitive status, disease duration, APOE genotype, and biomarker levels. The patterns of key network destructions parallel the tau and atrophy patterns of the AD subgroups essentially. Interruption of key networks, in particular the default-mode network that is responsible for cognitive decline, is consistent in heterogenous $A D$ groups. $A D$ pathology is often associated with
\end{abstract}

karger@karger.com

(c) 2021 S. Karger AG, Basel

www.karger.com/dem

Karger" co-pathologies: cerebrovascular lesions, Lewy pathology, and TDP-43 proteinopathies. These mixed pathologies essentially influence the clinical picture of $A D$ and may accelerate disease progression. Unraveling the heterogeneity among the $A D$ spectrum entities is important for opening a window to pathogenic mechanisms affecting the brain and enabling precision medicine approaches as a basis for developing preventive and ultimately successful disease-modifying therapies for $A D$.

(c) 2021 S. Karger AG, Basel

\section{Introduction}

Alzheimer disease (AD), the most common form of dementia in adults that currently affects around 50 million people worldwide, was initially defined as a clinicopathological entity. In terms of neuropathology, $\mathrm{AD}$ is defined as a brain disease with deposition of $\beta$-amyloid $(A \beta)$ in extracellular $A \beta$ plaques and in the vasculature (cerebral amyloid angiopathy/CAA), neuritic plaques (a subset of senile plaques defined by the presence of hyperphosphorylated tau-protein/p-tau), and intraneuronal aggregation of $\mathrm{p}$-tau protein forming neurofibrillary tan- 
gles (NFTs). These changes are accompanied by early synaptic loss [1], immune-responsive microglia [2], reactive astroglia [3], neurovascular dysfunction [4], disruption of the blood-brain barrier [5], neuronal loss, and brain atrophy [6]. A neuropathological diagnosis of $\mathrm{AD}$ is made according to the updated National Institute of Aging/Alzheimer's Association (NIA-AA) "ABC" criteria [7]. This $\mathrm{ABC}$ score for $\mathrm{AD}$ neuropathological changes (ADNC) combines " $A$ " for the phase of amyloid plaques [8]; "B" for the NFT stages [9], and "C" for the CERAD neuritic plaque score [10]. These pathological changes, which involve brain regions and neuronal cell types following a characteristic pattern [9] as a result of selective cellular and regional vulnerability to pathological proteins and their spread through the brain [11], present clinically as progressive decline in cognition and other functions.

Classically, AD manifests as a progressive, multidomain amnestic syndrome, but individual patients show variant syndromes, which present major challenges for both diagnosis and monitoring of disease progression [12]. $\mathrm{AD}$ is a heterogenous, multifactorial continuum with several clinically and pathobiologically defined subtypes, currently referred to as Alzheimer clinical syndrome [13]. Its variability in age and clinical presentation is very well known, such as the clinical and morphological differences between early-onset $\mathrm{AD}(\mathrm{EOAD})$ that may occur on a monogenic basis [14] and the much more common and apparently sporadic late-onset $\mathrm{AD}$ (LOAD) $[15,16]$. EOAD is associated with a higher burden of ADNC and a higher rate of neocortical atrophy [17], but shows significant heterogeneity in cognitive presentation and patterns of atrophy/hypometabolism [18].

Regarding variation in the clinical presentation, different phenotypes based on consensus and accepted guidelines have been determined, which show that the amnestic syndrome [19] is more common than nonamnestic presentations [20], such as posterior cortical atrophy [21], logopenic primary progressive aphasia (LPPA) [22, $23]$, and the behavioral variant of $\operatorname{AD}[24,25]$, while the corticobasal syndrome (CBS) subtype of AD shows an atypical distribution of ADNC [26].

\section{Clinicopathological Diversity of AD Variants}

Recent progress in biomarker research and wider availability of postmortem data has enabled the identification of pathophysiologically defined subtypes of $\mathrm{AD}$, based on neuropathological, neuroimaging, and in vivo biomarker data. With the new definition of $\mathrm{AD}$ as a biologically defined spectrum, using the NIA-AA research framework [13], it is now possible to review the pathobiologically defined subtypes of AD [27]. Research consensus guidelines have been proposed for the intravitam biologically based categorization termed "ATN," which uses flexible combinations of in vivo biomarkers for $A \beta$ deposition (A), tau pathology (T), and neurodegeneration $(\mathrm{N})$; they include cerebrospinal fluid (CSF) or plasma biomarkers, PET, and functional and structural MRI. The biomarker profiles and categories of the Alzheimer continuum referring to ADNC have been summarized recently [28]. The risk of progressive cognitive deterioration differs considerably between the various subtypes: $\mathrm{A}-\mathrm{N}-<\mathrm{A}+\mathrm{N}-<\mathrm{A}+\mathrm{T}-\mathrm{N}+<\mathrm{A}+\mathrm{T}+\mathrm{N}+$, the $\mathrm{A}+\mathrm{T}-\mathrm{N}+$ cas es with the worst prognosis versus negative $(\mathrm{A}-\mathrm{T}-\mathrm{N}-)$ ones [29].

Several subtypes of AD have been proposed based on clinical, biological, and neuropathological features that have been evaluated in a systematic review and metaanalysis [27]. A seminal study of 889 brain autopsies classified 3 AD subtypes based on postmortem NFT density: typical AD with balanced NFT counts in neocortex and hippocampus (75\% of cases), hippocampal-sparing (HcSp) AD, with NFT counts predominantly in association cortices (11\%), and limbic-predominant (LP) AD, with NFT mainly involving the hippocampus (14\%) [30]. These neuropathological subtypes had also different clinical phenotypes, with different ages at onset and rates of progression. Patients with $\mathrm{HcSp}-\mathrm{AD}$ had earliest age at onset, a higher proportion of men, and progressed more quickly than typical $\mathrm{AD}$, while $\mathrm{LP}-\mathrm{AD}$ patients were older, had a higher proportion of women, and progressed more slowly. Disease duration of LP-AD and typical AD cases was similar, while the Mini-Mental State Examination (MMSE) was lowest in the HcSp form but did not differ from typical AD. The age at death for the LP form was highest, while patients with $\mathrm{HcSp}-\mathrm{AD}$ were youngest, reflecting that the latter is the most aggressive. NFT accumulation in the nucleus basalis of Meynert may underlie more widespread and severe cholinergic deficits in young-onset $\mathrm{AD}$, in particular with $\mathrm{HcSp}(\mathrm{p}=0.3)$ and typical AD $(\mathrm{p}<0.001)$ and APOEc4 allele $(\mathrm{p}=0.3)$ [31]. This is coherent with the possible contribution of TDP-43 pathology, hippocampal sclerosis, and the microtubuleassociated protein tau (MAPT) H1H1 genotype to LP$\mathrm{AD}$, factors related to atrophy restricted to the temporal lobes, older age, and slower disease progression. APOE 4 carriers more frequently had LP and typical $\mathrm{AD}$, whereas noncarriers more frequently had $\mathrm{HcSp}-\mathrm{AD}$. APOE 4 has 
Table 1. Major characteristics of Alzheimer disease (AD) subtypes [34]

\begin{tabular}{lccc}
\hline Characteristics & HcSp-AD & LP-AD & Typical AD \\
\hline Total, $n$ & $79(8.2 \%)$ & $85(8.9 \%)$ & $769(82.5 \%)$ \\
$\quad$ Females, $n$ & $47(59.5 \%)$ & $55(64.7 \%)^{*}$ & $520(67.5 \%)^{*}$ \\
Age & & & \\
$\quad$ At death & $76.3 \pm 8.6$ & $84.9 \pm 3.8^{*}$ & $81.3 \pm 9.2^{*}$ \\
$\quad$ At onset & $68.0 \pm 10.0^{*}$ & $73.8 \pm 6.4^{*}$ & $79.7 \pm 3.8^{* * *}$ \\
Disease duration & $7.4 \pm 3.6^{* *}$ & $4.8 \pm 2.6^{* * *}$ & $9.1 \pm 4.3^{*}$ \\
Mean final MMSE & $10(n=20)$ & $11.5(n=16)$ & $4.6(n=78)^{\mathrm{a}}$ \\
Median Braak NFT stage (range) & $4.5(2-5)$ & $4.5(3-5)$ & $5.6(5-6)$ \\
Cerebrovascular pathology, $n$ & $27(34.2 \%)^{\mathrm{b}}$ & $35(41.1 \%)$ & $286(36.4 \%)^{\mathrm{b}}$ \\
Lewy body pathology, $n$ & $19(24.9 \%)^{\mathrm{a}}$ & $3(3.5 \%)$ & $63(8.2 \%)$ \\
\hline
\end{tabular}

Means $\pm \mathrm{SD}, n(\%)$, or medians (ranges). MMSE, Mini-Mental State Examination; NFT, neurofibrillary tangles; HcSp-AD, hippocampal-sparing AD; LP-AD, limbicpredominant AD. ${ }^{*} \mathrm{p}<0.01,{ }^{* *} \mathrm{p}<0.001,{ }^{* * *} \mathrm{p}<0.001$, vs. HcSp-AD, ${ }^{a} \mathrm{p}<0.01$ vs. other forms, ${ }^{\mathrm{b}} \mathrm{p}<0.01$ vs. LP-AD. been associated with a lower volume in the hippocampus and adjacent regions $[32,33]$. Therefore, APOE\&4 negativity may increase resistance of the hippocampus against tau pathology. Vascular co-pathology (ranging from 16 to $36 \%$ ) was highest in the LP and lowest in the HcSp cases, while Lewy co-pathology was lowest in the latter type. These data were largely confirmed by another study in 933 autopsy cases of $\mathrm{AD}$, all with neuritic Braak stage $>4$ (Table 1 [34]). Typical AD in our cohort was more frequent than in the Mayo series ( 82.5 vs. $75 \%$ ), while the other 2 subtypes were slightly less frequent. MA-AD was not considered in this study. The LP-AD cases shared some morphological characteristics with primary age-related tauopathy (PART) [35], although this was not confirmed later, which emphasizes significant pathological differences versus LP-AD and suggests that it may not merely be a variant of $\mathrm{AD}$ [36]. PART, predominantly involving people aged $>85$ years and associated with mildto-moderate cognitive impairment $[37,38]$, is morphologically characterized by tau pathology restricted to the medial temporal lobe (Braak stages $0-\mathrm{IV}$ ), relative absence of amyloid (Thal $A \beta$ phases $0-2$ ), total absence of neuritic plaques, and rare CAA [39]. neurofibrillary changes in PART are identical to those in classical AD, with both 3 and 4 repeat ( $3 \mathrm{R}$ and $4 \mathrm{R}$ ) tau isoforms [40]. PART is considered either a prodromal form or a subtype of $\mathrm{AD}$ [41]. MAPT $\mathrm{H} 1 \mathrm{H} 1$ genotype frequency is high in both PART and LP-AD, and similar to typical AD, while the APOE\&4 carrier state is low in PART [42]. In PART, the lack of $A \beta$ oligomers is responsible for less severe tau aggregation and, thus, for lower Braak stages and less cognitive impairment since $A \beta$ oligomers have been shown to potentiate tau aggregation by promoting tau seed uptake [43]. While the incidence of classical AD increases from the 7th to the 9th decade and shows a mild decrease later, the frequency of PART increases after 85 years of age [44].

In the Vienna series, the proportion of females was highest in typical $\mathrm{AD}(67.5 \%)$ and lowest in HcSp-AD (59.5\%). Similar to the Mayo series, age at disease onset and death was significantly higher in LP than in typical $\mathrm{AD}(p<0.01)$, and significantly lower in HcSp-AD. Final MMSE scores were lowest in typical $\mathrm{AD}$, and they did not essentially differ between the 2 other types, whereas in the Mayo series, the final MMSE scores were lowest in the $\mathrm{HcSp}$ form and slightly higher in typical AD cases. Among co-pathologies, cerebrovascular lesions were more frequent in our cohort (34.2-41.1\%) and most frequent in LP-AD cases. Lewy body (LB) pathology was highest in $\mathrm{HcSp}$ forms (24\%, due to the frequent association of dementia with LB), and much lower in the 2 other subtypes (3.5 and $8.2 \%$, respectively), thus less frequent than in the Mayo series.

\section{Neuroimaging Findings in AD Subtypes}

The 3 AD subtypes, based on the distribution of NFTs and corresponding brain atrophy, have also been identified by neuroimaging studies using structural MRI [4548]. Brain atrophy corresponds well with NFT topography, and amnestic and nonamnestic symptoms correspond to regions of neuronal injury and correlate to neuronal hypometabolism [30, 45, 49]. 
Fig. 1. Main factors and characteristics of the 4 main subtypes of AD. AD, Alzheimer's disease; NFT, neurofibrillary tangle; $\mathrm{WMH}$, white matter hyperintensity; CAA, cerebral amyloid angiopathy.
- Relative frequency $55 \%$

Typical AD

- Balanced NFT counts in hippocampus and association cortex

- Multidomain amnestic syndrome

- Low final MMSE scores

- High A $\beta$ PET in occipital cortex, less in medial temporal and parietal cortex

- High burden of WMHs, CAA frequent

- APOE $\varepsilon 4$ genotype frequent

- Increased Lewy and TDP-43 pathologies

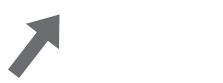

Hippocampal-sparing $A D$

- Relative frequency $17 \%$

- NFT counts preliminary in neocortex

- Higher rate of neocortical atrophy

- Young onset/death

- Male sex

- Nonamnestic syndrome

- Faster disease progression

- Most aggressive form

- High educational level/cognitive reserve

- APOE \&4 noncarriers

- Low burden of WMHs

- Marked A $\beta$ PET binding in frontal and parietal cortex

- Less frequent Lewy and TDP-43 pathologies

Limbic-predominant AD
- Relative frequency $21 \%$
- NFT counts predominantly in hippocampus
- More severe atrophy of medial temporal
lobe
- Older age at onset/death
- Female sex
- Amnestic syndrome
- Slow disease progression
- Greater A 3 PET binding in frontal and
parietal cortex
- Higher burden of WMHs
- Overloaded cognitive reserve
- APOE $\varepsilon 4$ and MAPT H1H1 genotypes frequent
- Hippocampal sclerosis
- TDP-43 proteinopathy frequent
- Less CAA, more hypertensive arteriopathy

Recent MRI studies have consistently identified 4 categories of brain atrophy: both hippocampal and cortical atrophy, hippocampal only, cortical only, and no or minimal gray matter atrophy, which, however, shows comparable clinical severity [50-55]. Compared with the other 3 subtypes, MA-AD showed an intermediate age at onset. Despite not presenting with significant atrophy but having AD CSF biomarker levels (CSF p-tau increased and $\mathrm{A} \beta$ decreased), this group fulfilled the diagnostic criteria for $\mathrm{AD}$ but had a slow rate of disease progression. This was concordant with other studies $[49,56,57]$.

Because the 4 subtypes did not differ substantially in disease duration, the age at the time of MRI was the lowest in HcSp-AD and the highest in typical and LP-AD, with MA-AD in between, although timing of MRI studies differed considerably in postmortem studies [27, 48]. Among $175 \mathrm{AD}$ patients and 81 healthy controls from the ADNI-1 study, $50.9 \%$ were typical AD, $17.1 \%$ LP-AD, 
16.1\% HcSp-AD, and 15.4\% MA-AD [52]. Another MRI study of brain atrophy subtypes that may predict longterm cognitive decline in $\mathrm{AD}$ distinguished $\mathrm{HcSp}$, cortical, homogenous atrophy, and "no evidence of brain atrophy" subtypes. The MA-AD subtype was associated with an increased risk of developing the clinical $\mathrm{AD}$ over time [58]. A recent study using MRI, tau, and A $\beta$ PET based on cortical atrophy revealed 3 subtypes of $A D$ : medial temporal (53\%), parietal dominant (22\%), and diffuse atrophy (23\%), while $A \beta$ deposition did not differ across the subtypes [59], suggesting a typical pattern of tau spread, with NFTs developing in the entorhinal cortex and then processing to the association cortices; the MA form would be the earliest presentation, which progresses via the LP-AD finally to typical AD (Fig. 1). However, there is growing evidence supporting the alternative "distinct subtype hypothesis," which suggests actual heterogeneity of disease progression [30, 60-62].

Studies of the tau PET patterns in $260 \mathrm{~A} \beta$-positive patients with mild cognitive impairment (MCI) or dementia revealed the greatest tau load in HcSp-AD (frontoparietal predominant) and typical $\mathrm{AD}$ (temporal predominant), while LP patients showed excessively higher entorhinal tau load. These data suggest that structural MRI can be used to identify biologically and clinically meaningful subtypes of AD [63]. Tau pathology is closely associated with sites of neurodegeneration $[24,64,65]$. Typical AD had the most prominent and greater white matter hyperintensity burden, which may be due to wallerian degeneration induced by cortical tau pathology [66], small vessel disease, or both $[67,68]$. The HcSp patients had often nonamnestic disease presentation and most rapid cognitive decline. Recent studies showed that flortaucipir PET reflects neuritic Braak stages IV or greater neuropathology, thus being a reliable biomarker of underlying NFT pathology [69].

Tau pathology and neurodegeneration at the molecular level can be sufficient to disrupt key brain networks, which could induce symptoms in the absence of overt brain atrophy of the medial temporal lobe in MA-AD that shows memory impairment comparable to LP and typical $\mathrm{AD}[48,52,61]$. This is supported by the finding of reduced metabolism in the parietal cortex in these patients [70]. The devastation of the medial temporal lobe may explain memory impairment $[45,47,49,56,57,61]$. Distinct signature patterns of network destruction, which parallel the NFT and atrophy pattern of the 4 subtypes, have been demonstrated, and are consistent with isocortical Braak stage $\mathrm{V}$ [52]. The cortical areas of the defaultmode network are involved in typical, LP-, and MA-AD, while 3 major networks in posterior brain regions (frontoparietal, visual, and default-mode networks) are affected in MA-AD [52]. Hence, disruption of the defaultmode network is a consistent damage in heterogenous $\mathrm{AD}$ groups [47, 71]. These findings support the distinct subtype hypothesis, with pathology spreading through the brain in a different manner in these subtypes, as opposed to the staging hypothesis $[30,52,60]$.

Typical AD had higher $A \beta$ plaque counts in occipital regions than LP-AD [30], which showed greater A $\beta$ PET binding in frontal and parietal cortices than typical AD [46]. However, in contrast to specific tau accumulation and brain atrophy patterns among $\mathrm{AD}$ variants, $\mathrm{A} \beta \mathrm{ac}-$ cumulation appears rather diffuse and is similar across groups, with the exception of the MA group [72, 73]. Since $A \beta$ deposition develops decades prior to the first signs of cognitive impairment [74], it might be possible that it initially develops differentially in AD variants before reaching a plateau at a relatively early stage [11]. Autopsy studies have found that tau pathology is more closely related to atrophy and clinical symptoms than $A \beta$ pathology [75]. However, the synergism between $A \beta$ deposition and NFT neurodegeneration may be a better predictor of cognitive decline or disease progression than either pathology alone [76]. The revised NIA-AA guidelines for the pathological diagnosis of $A D$, which take $A \beta$ Thal phase, neuritic Braak stage, and co-occurrence of pathological tau within $A \beta$-containing neuritic plaques into account, are now a widely accepted guide for assessing ADNC severity [7]. CAA seemed to make a stronger contribution to HcSp- and MA-AD, whereas hypertensive arteriopathy had a stronger influence on typical AD and LP-AD.

$\mathrm{HcSp}-\mathrm{AD}$ cases have been found to have the highest level of education, while MA-AD patients had the lowest, suggesting that high education may be one of the factors protecting the hippocampus and thus contributing to the fact that LP-AD may be unmasked and may contribute more to the clinical manifestation of the disease. The theory of cognitive reserve suggests that education is beneficial for the brain by forming more efficient and pathology-resistant networks; it may act as surrogate for or act in synergy with cognitive or social engagement [77, 78]. This suggests that people with higher reserve can cope with brain pathology better [79]. More severe neurodegeneration and more aggressive disease progression in HcSp-AD [30, 36, 47, 51, 80], and the absence of hippocampal brain atrophy in the MA form, which has a lower level of reserve, support this hypothesis [27]. A higher cognitive reserve has been associated with lower cortical 
Table 2. Atypical variants of late- and early-onset Alzheimer disease (modified from [11])

\begin{tabular}{lllll}
\hline AD subtype & Clinical presentation & Pathology & Progression & CSF A $\beta$ and tau levels \\
\hline Typical LOAD (50-75\%) & $\begin{array}{l}\text { Amnestic/ } \\
\text { nonamnestic }\end{array}$ & $\begin{array}{l}\text { Both hippocampal and } \\
\text { cortical (“both impaired”) }\end{array}$ & Typical AD & Typical AD \\
\hline Limbic-predominant LOAD (15-35\%) & Amnestic & Medial-temporal lobe ("hippocampal”) & Slower & Similar \\
\hline Hippocampal-sparing LOAD (10-25\%) & Nonamnestic & “Cortical," medial temporal lobe sparing & Faster & Similar \\
\hline Minimal-atrophy LOAD (10-17\%) & Unclear & Minimal & Slowest & A $\uparrow$, tau $\downarrow$ \\
\hline Typical EOAD (75\%) & $\begin{array}{l}\text { Amnestic/ } \\
\text { nonamnestic }\end{array}$ & Hippocampal and cortical & Typical AD & Similar to typical AD \\
\hline Atypical EOAD (25\%) & Nonamnestic & Posterior cortical & Faster & Similar to typical AD \\
\hline
\end{tabular}

$\mathrm{AD}$, Alzheimer disease; $\mathrm{LOAD}$, late-onset $\mathrm{AD}$; $\mathrm{EOAD}$, early-onset $\mathrm{AD}$.

tau-PET binding, which has been interpreted as brain reserve/higher cognitive reserve possibly contributing to avoid tau aggregation [81].

A recent study using diffusion tensor imaging and latent Dirichlet allocation for the white matter found a different composition profile of latent impairment factors, which indicated heterogeneity and subtypes of $\mathrm{AD}$ through white matter impairment factors. The latent factors have distinct impact on cognitive decline: for executive function, the temporofrontal compared with the parietal factor, while the long-fiber factor was most associated with executive function decline. Three factors (temporofrontal, parietal, and long-fiber factor) showed almost equal effect on the memory decline rate. Thus, white matter impairment factors contribute to investigating heterogeneity and subtypes of $\mathrm{AD}[82]$. Another recent study of 1,677 dementia cases from the National Alzheimer's Coordinating Center Registry separated "tangle-intensive" and "plaque-intensive" cases from "classic $\mathrm{AD}$," which indicates that neuropathology does not directly reflect current consensus recommendations [83].

The APOE genotype was found to affect the AD subtype. Specifically, APOE\&4 was more associated with the long-fiber bundle factor and APOE\&2 with temporofrontal factor, confirming the heterogeneity of AD. Genetic research in AD subtypes also focuses on the MAPT H1H1 genotype, which is increased in LP-AD compared to the others $[30,36]$. However, another study reported its highest frequency in typical AD, followed by the 2 others [48]. These findings suggest that the MAPT $\mathrm{H} 1 \mathrm{H} 1$ genotype may be important for the medial temporal predilection of tau pathology. The proportion of APOE 4 is elevated in amnestic but not in aphasic manifestations of $\mathrm{AD}$. It is an anatomically selective risk factor that increases the vulnerability in memory-related medial-temporal areas rather than language-related areas [84].

A recent meta-analysis of 24 studies calculated the pooled frequency of the $4 \mathrm{AD}$ subtypes. Typical AD, characterized by tau pathology and atrophy in both hippocampus and association cortex, is the most frequent subtype, with a pooled frequency of 55\%. LP-, HcSp- and MA-AD had a pooled frequency of 21,17 , and $15 \%$, respectively [27]. These data depended on the algorithm used for biological and nonbiological subtyping, such as age at onset and death, disease duration, education years, MMSE, clinical dementia rating, APO status, and CSF biomarkers (see Table 2 [11]).

\section{Neuropathology of Other Clinical AD Variants}

Pathologically proven $\mathrm{AD}$ shows a broad spectrum of clinical manifestations beyond the classical progressive amnestic-predominant syndrome. Clinicopathological studies have identified a number of atypical nonamnestic syndromes generally referred to as atypical or focal $\mathrm{AD}$ $[12,85,86]$. The major atypical variants of EOAD and LOAD are presented in Table 2. More recent studies have defined a larger variety of atypical clinical syndromes, including LPPA [22, 23, 87], posterior cortical atrophy [21], CBS $[26,88-90]$, and behavioral/dysexecutive syndrome resembling the behavioral variant of frontotemporal dementia (bvFTD) [24, 25], and a nonamnestic AD with TDP-43 pathology [20]. Atypical clinical variants of AD often show a younger age at onset and fewer associations with the APOE 4 genotype compared to typical amnestic 
forms $[30,91]$. Recent studies in a large postmortem series investigated the clinical relevance of regional NFT pathology with regard to various clinical variants of $\mathrm{AD}$ and domain-specific clinical representations [92]. Individuals with LPPA showed significantly higher NFT density in the superior temporal gyrus relative to those with an amnestic syndrome and to other atypical variants, while those with CBS revealed higher NFT density in the perirolandic cortices, namely the primary motor and somatosensory cortex, but less in the superior temporal cortex. However, the neuritic Braak stage and the Thal amyloid phase were similar to amnestic AD. ADNC had a predilection for left language regions that showed the most severe atrophy in primary progressive aphasia [93]. The CBS subtype of AD had greater neuronal loss in the substantia nigra, which may contribute to parkinsonism that is not a typical feature of classic $\mathrm{AD}$ [26]. In a series of pathologically confirmed nonamnestic cases, $44 \%$ had typical AD pathology and were TDP-43 positive, which, however, did not essentially influence the clinical and anatomical features of $\mathrm{AD}[20]$. Both behavioral and dysexecutive $\mathrm{AD}$ are distinguished by temporoparietal-predominant atrophy, but frontotemporal lobar degeneration-tau and -TDP-43 pathology occur in various degrees and several co-pathologies, such as CAA, dementia with LB, and argyrophilic grain disease [24], whereas others found a higher NFT burden in the subiculum [92]. In the Mayo series, posterior cortical atrophy, LPPA, and bvFTD variant were more common in HcSp-AD than in LP-AD and typical $\mathrm{AD}[30,46,94]$, specific functional consequences of regional tau/NFT accumulation, the mapping of which may increase the understanding of functionalclinical manifestations of AD.

The neurobiological features underlying $\mathrm{AD}$ subtypes are largely unknown and cannot be explained by $\mathrm{A} \beta$ pathology, because the distribution of $\mathrm{A} \beta$ PET retention is virtually the same in all subtypes [72]. MA-AD is less affected by tau pathology but also $A \beta$ positive. This suggests that multiple subtypes are in reality one of the same AD continuum of a single disease type [24].

\section{More Indicators of AD Heterogeneity}

The original ADNI (Alzheimer's Disease Neuroimaging Initiative) data considered 5 diagnostic groups in a total 658 patients (AD: $n=110$, late MCI: $n=133$, early MCI: $n=148$, significant memory concern: $n=94$, and cognitive normal: $n=173$ ). Clustering the cohort by applying a novel big data analysis strategy [95], a recent study suggested a different set of 6 subtypes which present different clinical patterns and potential biomarkers: "healthy" ( $n=121)$, "affective MCI" $(n=101)$, "anosognosia dementia" $(n=54)$, "worried well" $(n=148)$, "uncompensated MCI" $(n=136)$, and "insightful dementia" $(n=98)$ [96].

A study comparing cognitive disorders with MRI and PET findings in 146 EOAD subjects and 395 age-matched controls differentiated 4 clusters: cluster 1 (memory-predominant impairment) presented atrophy/hypometabolism in medial/lateral temporal, lateral parietal, and posterior cingulate, and cluster 2 (memory/visuospatial predominant) involved medial temporal, temporoparietal, and frontal cortices; cluster 3 (memory, language, and executive function) and cluster 4 (globally impaired) both showed atrophy and hypometabolism throughout the brain. Cluster differences between visuospatial/executive domains were significant, indicating heterogeneity in cognitive presentation among amnestic EOAD subjects and patterns of atrophy/hypometabolism in each cluster in agreement with the observed cognitive phenotypes, suggesting phenotypic variation [18].

Another study classified 4,050 people with LOAD into 6 groups based on their cognitive functioning in 4 domains: memory, executive and visuospatial functioning, and language, and then genetic data were used to find biological differences among cognitively defined subgroups [97]. The largest group (39\%) had fairly similar scores in all 4 domains, followed by those (27\%) who had significantly lower memory scores; smaller groups had substantially lower language scores $(13 \%)$, others lower visuospatial functioning scores (12\%) and lower executive functioning scores (3\%), while another $6 \%$ had 2 domains that were substantially lower than the other scores. Single nucleotide polymorphisms (SNPs; $n=33$ ) were found throughout the genome, where the genetic association was very strong for 1 of the 6 subgroups, indicating that $\mathrm{AD}$ is not a single homogenous condition.

\section{The Challenges of Co-Pathologies}

AD pathology rarely occurs in isolation, and community-based neuropathological studies have shown that complex constellations of underlying pathologies may lead to cognitive decline. The number of possible combinations or comorbidities increases in the aging brain, and frequently mixed pathologies ensue $[38,44,67,98-$ 100]. Patients with ADNC often have concomitant pathologies, such as cerebrovascular disease (CVD), LB pa- 
thologies, and/or TDP-43 proteinopathy $[101,102]$. In a consecutive series of 1,700 elderly demented patients and those with the clinical diagnosis of $\mathrm{AD}, \mathrm{ADNC}$ were present in $82.9 \%$ of all demented and in $92.8 \%$ of those with the clinical diagnosis of $\mathrm{AD}$, but only half of them (41.1 and 47.6\%, respectively) showed pure ADNC (ABC $3 / 3 / 3$ ), while all the others were either atypical AD forms or subtypes (including PART; 7 and 6\%, respectively) or showed additional CVD, cerebral hemorrhage, LB, or other mixed pathologies, while vascular dementia in this cohort accounted for only 10.7 and 3.3\%, respectively, and other non- $\mathrm{AD}$ pathologies were seen in 17 and $7.2 \%$, respectively [103]. An earlier study of AD in clinical trials revealed pure ADNC in only $31 \%$ and multiple pathologies in $63 \%$, while no pathological changes were seen in $1 \%$ [104]. A review of 12 community-based studies with 3,574 patients, irrespective of the clinical symptoms, reported a frequency of ADNC between 19 and 67\%, of LB pathologies between 6 and 39\%, of vascular pathologies between 28 and 70\%, of TDP-43 proteinopathy between 19 and $75 \%$, of hippocampal sclerosis between 3 and $13 \%$, and, finally, of mixed pathologies between 8 and 70\% [99]. Data from the Religious Orders Study and Memory and Aging Project cohort showed that about $50 \%$ of persons diagnosed with probable AD had mixed pathologies $(\mathrm{AD}$, dementia with $\mathrm{LB} /$ Parkinson disease, and CVD). Among 447 patients with probable AD, only $3.13 \%$ showed pure $\mathrm{ADNC}, 27.3 \% \mathrm{AD}+\mathrm{CVD}+$ other, $3 \% \mathrm{AD}+\mathrm{CVD}, 7.6 \% \mathrm{AD}+$ other degenerative lesions, and $47 \% \mathrm{AD}+\mathrm{CVD}+$ other neurodegenerative changes [100]. Lower cognitive reserve and small-vessel disease in strategic white matter areas [62] could increase network vulnerability to tau pathologies and neurodegeneration in MA-AD [105]. Similar comorbidities in 1,153 patients with the clinical diagnosis of $\mathrm{AD}$ were reported recently [106], and among 673 autopsy cases, 301 of which were demented, the majority of the demented cohort showed mixed pathologies [67]. LP age-related TDP-43 encephalopathy neuropathological change (LATE-NC) was present in approximately $57 \%$ of AD cases and was associated with accelerated cognitive decline and accelerated disease progression [107]. The strong association between hippocampal sclerosis and TDP-43 has been confirmed by the fact that TDP-43 is increased in typical and LP-AD compared to HcSp-AD $[30,94]$. On the other hand, among 172 autopsy-proven $\mathrm{AD}$ cases, $19 \%$ were classified as nonamnestic, $69 \%$ of which had typical ADNC and 44\% were TDP-43+; $31 \%$ were $\mathrm{HcSp}-\mathrm{AD}$, of which $36 \%$ were TDP-43+, but no LP$\mathrm{AD}$ were found [20]. However, clinical presentation of
AD seems to be driven by subtype and not by TDP- 43 [94]. This is strengthened by a recent study of 46 autopsy-confirmed $\mathrm{AD}$ cases, $63 \%$ of which exhibited LATE$\mathrm{NC}(\mathrm{AD}+)$ and a higher burden of p-tau (AT8). No association between AT8 with LATE-NC score was revealed, but the higher intensity of AT8 pathology indicated possible progression of the disease compared to $\mathrm{AD}$ cases, whereas the presence of LATE-NC was not associated with differences in cognitive scores in $\mathrm{AD}$.

A recent clinicopathological study using data from the National Alzheimer's Coordination Center showed that 1,854 participants had a clinical diagnosis of $\mathrm{AD}$ and ADNC at autopsy (confirmed AD); 204 with a clinical AD diagnosis had no ADNC (AD mimics), and 253 participants with no clinical AD diagnosis had ADNC (unidentified $\mathrm{AD}$ ). Compared to confirmed AD cases, AD mimics (e.g., frontotemporal lobar degeneration-tau, hippocampal sclerosis, and cerebrovascular pathology) had less severe cognitive impairment, while unidentified $\mathrm{AD}$ participants displayed more parkinsonian signs, depression, and behavioral problems. This study emphasizes the importance of developing a complete panel of biomarkers as a tool to promote clinical diagnosis, as clinical phenotypes that are typically associated with diseases other than $\mathrm{AD}$ may result in inaccurate diagnosis [108].

A comparative study of clinical and neuropathological diagnoses of $\mathrm{AD}$ in 3 epidemiological samples reported a sensitivity of probable AD of $93 \%$ [109]. Meta-analysis of 20 (out of 1,189) studies to distinguish autopsy-verified $\mathrm{AD}$ from other dementias or healthy controls showed a sensitivity of $85.4 \%$ (95\% CI $80.8-90 \%)$ and a specificity of $77.7 \%$ (95\% CI 70.2-85.1\%). Values were higher for neuroimaging procedures and slightly lower for CSF biomarkers, while the combination of both resulted in higher values [110]. These data are related to 2 factors: (1) the various subtypes or variants of $\mathrm{AD}$, as described above, and (2) the high frequency of comorbidities in elderly persons that may cause difficulties in the clinical diagnosis of AD. A recent study showed that antemortem ${ }^{18} \mathrm{~F}$ flortaucipir PET is able to identify the underlying presence of tau (NFT) pathology at the B3 level and a high level of ADNC per the NIA-AA criteria consistent with a neuropathological diagnosis of $\mathrm{AD}$ [111].

In summary, recent community-based neuropathological studies have demonstrated that the complex constellations of the underlying pathologies may lead to cognitive decline, and that the number of possible combinations/comorbidities increases in the aging brain [38]. All these concomitant pathologies may be especially harmful to individuals with low cognitive reserve such as patients 
with MA-AD. Total burden of comorbid pathological abnormalities, rather than any single lesion, is the most relevant determinant of cognitive impairment, often despite a clinical diagnosis of "only" AD [112].

\section{Conclusions and Perspectives}

$\mathrm{AD}$ is a multifactorial disorder, the clinical and morphological heterogeneity of which is documented by several subtypes that have a distinct signature of network disruptions associated with their atrophy pattern probably reflecting the differential spread of NFT pathology and different vulnerability pattern of affected brain regions. These facts are substantially related to $3 \mathrm{important}$ factors, that is, risk factors, protective factors, and concomitant pathologies. The balance between risk and protective factors determines brain cellular and regional vulnerability, which contributes to differential spatial manifestation of pathologies and potential disease-relevant lesion patterns, and eventually leads to divergent clinicopathological presentations of AD. The severity dimension corresponds to the "N" category in the new A/T/N classification for biomarkers [113], indicating that the Alzheimer clinical syndrome includes several pathobiologically defined entities or subtypes, which are clinically and morphologically different. This heterogeneity is due to multiple pathogenic factors, for example, "upstream" genotypes causing comorbidities, "downstream" disease-modifying gene variants (MAPT, H2 haplotype, APOE, TREM, and GRN variants) which induce misfolding tau, A $\beta$, TDP-43, and others, the synergetic or additive action of which results in various disease phenotypes [114]. It is possible that factors such as brain resilience may help compensating for these pathologies up to a certain level. However, a combination of risk factors may knock out these compensatory effects. In the absence of these risk factors, brain resistance mechanisms may more efficiently preserve vulnerable cell populations and brain regions, and reduce the vulnerability of key brain networks. It has been suggested that individuals should be identified who have connectivity patterns resistant to the initiation or spread of neurodegeneration. This may be an important factor underlying incomplete penetrance of genetic mutations, differential gene expression across brain regions, or other substrates such as association with minimal or no comorbid abnormalities [115]. Furthermore, compensation strategies could be used in $\mathrm{HcSp}$ - and MA-AD, because the learning and encoding capacity in these subtypes is partially spared
[61]. The prevalence of biological $\mathrm{AD}$ with its various subtypes and variants is greater than clinical probable $\mathrm{AD}$ at any age, in particular at $85+$ years of age [13]. Therefore, recent studies have shown that different individuals with typical $\mathrm{AD}$ may have distinct biochemical features of tau that contribute to the clinical heterogeneity of $\mathrm{AD}$ [116]. These facts and the increasing incidence of AD illustrate its consequences on public health. In order to increase the sensitivity and specificity of the new ATN biomarker system, more extensive clinicopathological studies in well-defined populations will be necessary, with postmortem studies using the updated NIAAA criteria. Neuropathological studies should use a wide range of molecular pathological methods and should evaluate multiple CNS regions. An optimal and less costeffective strategy would be to screen specifically neurodegeneration-related proteins and to examine their cross reactions. Similar proposals have recently been published for validating the criteria for the diagnosis of $4 \mathrm{R}$ tauopathies [117]. Therefore, interdisciplinary studies to improve our knowledge about the pathogenesis of the heterogeneous manifestation of $\mathrm{AD}$ and to promote methods for its early diagnosis as the basis for further preventive and successful disease-modifying therapeutic measures are urgently needed.

Published in Celebration of the 30th Anniversary of the inception of Dementia and Geriatric Cognitive Disorders 1990-2020.

\section{Acknowledgment}

The author thanks Mr. Erich Mitter-Ferstl, PhD, for reference research and secretarial work.

\section{Disclosure Statement}

The author has no conflict of interest to declare.

\section{Funding Sources}

The study was supported by the Society for Support of Research in Experimental Neurology, Vienna, Austria. No other funding or sponsoring was involved in research and compilation of this manuscript. 


\section{References}

1 Chen MK, Mecca AP, Naganawa M, Finnema SJ, Toyonaga T, Lin SF, et al. Assessing synaptic density in Alzheimer disease with synaptic vesicle glycoprotein $2 \mathrm{~A}$ positron emission tomographic imaging. JAMA Neurol. 2018 Oct; 75(10):1215-24.

2 Keren-Shaul H, Spinrad A, Weiner A, Matcovitch-Natan O, Dvir-Szternfeld R, Ulland TK, et al: A unique microglia type associated with restricting development of Alzheimer's disease. Cell. 2017;169:1276-1290.e17.

3 Carter SF, Herholz K, Rosa-Neto P, Pellerin L, Nordberg A, Zimmer ER. Astrocyte biomarkers in Alzheimer's disease. Trends Mol Med. 2019 Feb;25(2):77-95.

4 Kisler K, Nelson AR, Montagne A, Zlokovic BV. Cerebral blood flow regulation and neurovascular dysfunction in Alzheimer disease. Nat Rev Neurosci. 2017 Jul;18(7):419-34.

5 Nation DA, Sweeney MD, Montagne A, Sagare AP, D’Orazio LM, Pachicano M, et al. Blood-brain barrier breakdown is an early biomarker of human cognitive dysfunction. Nat Med. 2019 Feb;25(2):270-6.

6 Pini L, Pievani M, Bocchetta M, Altomare D, Bosco P, Cavedo E, et al. Brain atrophy in Alzheimer's Disease and aging. Ageing Res Rev. 2016 Sep;30:25-48.

7 Montine TJ, Phelps CH, Beach TG, Bigio EH, Cairns NJ, Dickson DW, et al.; National Institute on Aging; Alzheimer's Association. National Institute on Aging-Alzheimer's Association guidelines for the neuropathologic assessment of Alzheimer's disease: a practical approach. Acta Neuropathol. 2012 Jan; 123(1):1-11.

8 Thal DR, Rüb U, Orantes M, Braak H. Phases of A beta-deposition in the human brain and its relevance for the development of AD. Neurology. 2002 Jun;58(12):1791-800.

9 Braak H, Braak E. Neuropathological stageing of Alzheimer-related changes. Acta Neuropathol. 1991;82(4):239-59.

10 Mirra SS, Hart MN, Terry RD. Making the diagnosis of Alzheimer's disease. A primer for practicing pathologists. Arch Pathol Lab Med. 1993 Feb;117(2):132-44.

11 Mrdjen D, Fox EJ, Bukhari SA, Montine KS, Bendall SC, Montine TJ. The basis of cellular and regional vulnerability in Alzheimer's disease. Acta Neuropathol. 2019 Nov;138(5): 729-49.

12 Warren JD, Fletcher PD, Golden HL. The paradox of syndromic diversity in Alzheimer disease. Nat Rev Neurol. 2012 Aug;8(8):451-64.

13 Jack CR Jr, Therneau TM, Weigand SD, Wiste HJ, Knopman DS, Vemuri P, et al. Prevalence of biologically vs clinically defined Alzheimer spectrum entities using the National Institute on Aging-Alzheimer's Association Research Framework. JAMA Neurol. 2019 Jul;76(10): 1174-83.
14 Ryan NS, Rossor MN. Defining and describing the pre-dementia stages of familial Alzheimer's disease. Alzheimers Res Ther. 2011 Sep;3(5):29.

15 Tellechea P, Pujol N, Esteve-Belloch P, Echeveste B, García-Eulate MR, Arbizu J, et al. Early- and late-onset Alzheimer disease: are they the same entity? Neurologia. 2018 May;33(4): 244-53.

16 Zhu XC, Tan L, Wang HF, Jiang T, Cao L, Wang C, et al. Rate of early onset Alzheimer's disease: a systematic review and meta-analysis. Ann Transl Med. 2015 Mar;3(3):38.

17 Marshall GA, Fairbanks LA, Tekin S, Vinters HV, Cummings JL. Early-onset Alzheimer's disease is associated with greater pathologic burden. J Geriatr Psychiatry Neurol. 2007 Mar;20(1):29-33.

18 Phillips ML, Stage EC Jr, Lane KA, Gao S, Risacher SL, Goukasian N, et al.; Alzheimer's Disease Neuroimaging Initiative. Neurodegenerative patterns of cognitive clusters of early-onset Alzheimer's disease subjects: evidence for disease heterogeneity. Dement Geriatr Cogn Disord. 2019;48(3-4):131-42.

19 Dubois B, Feldman HH, Jacova C, Hampel H, Molinuevo JL, Blennow K, et al. Advancing research diagnostic criteria for Alzheimer's disease: the IWG-2 criteria. Lancet Neurol. 2014 Jun;13(6):614-29.

20 Sahoo A, Bejanin A, Murray ME, Tosakulwong N, Weigand SD, Serie AM, et al. TDP43 and Alzheimer's disease pathologic subtype in non-amnestic Alzheimer's disease dementia. J Alzheimers Dis. 2018;64(4): 1227-33.

21 Crutch SJ, Schott JM, Rabinovici GD, Murray M, Snowden JS, van der Flier WM, et al.; Alzheimer's Association ISTAART Atypical Alzheimer's Disease and Associated Syndromes Professional Interest Area. Consensus classification of posterior cortical atrophy. Alzheimers Dement. 2017 Aug;13(8):870-84.

22 Ahmed S, de Jager CA, Haigh AM, Garrard P. Logopenic aphasia in Alzheimer's disease: clinical variant or clinical feature? J Neurol Neurosurg Psychiatry. 2012 Nov;83(11): 1056-62.

23 Spinelli EG, Mandelli ML, Miller ZA, SantosSantos MA, Wilson SM, Agosta F, et al. Typical and atypical pathology in primary progressive aphasia variants. Ann Neurol. 2017 Mar;81(3):430-43.

24 Ossenkoppele R, Pijnenburg YA, Perry DC, Cohn-Sheehy BI, Scheltens NM, Vogel JW, et al. The behavioural/dysexecutive variant of Alzheimer's disease: clinical, neuroimaging and pathological features. Brain. 2015 Sep; 138(Pt 9):2732-49.

25 Rascovsky K, Hodges JR, Knopman D, Mendez MF, Kramer JH, Neuhaus J, et al. Sensitivity of revised diagnostic criteria for the behavioural variant of frontotemporal dementia. Brain. 2011 Sep;134(Pt 9):2456-77.
26 Sakae N, Josephs KA, Litvan I, Murray ME, Duara R, Uitti RJ, et al. Clinicopathologic subtype of Alzheimer's disease presenting as corticobasal syndrome. Alzheimers Dement. 2019 Sep;15(9):1218-28.

27 Ferreira D, Nordberg A, Westman E. Biological subtypes of Alzheimer disease: A systematic review and meta-analysis. Neurology. 2020 Mar;94(10):436-48.

28 Jellinger KA. Towards a biological definition of Alzheimer disease. Int J Neurol Neurother. 2020;7:95.

29 Knopman DS, Haeberlein SB, Carrillo MC, Hendrix JA, Kerchner G, Margolin R, et al. The National Institute on Aging and the Alzheimer's Association Research Framework for Alzheimer's disease: Perspectives from the Research Roundtable. Alzheimers Dement. 2018 Apr;14(4):563-75.

30 Murray ME, Graff-Radford NR, Ross OA, Petersen RC, Duara R, Dickson DW. Neuropathologically defined subtypes of Alzheimer's disease with distinct clinical characteristics: a retrospective study. Lancet Neurol. 2011 Sep;10(9):785-96.

31 Hanna Al-Shaikh FS, Duara R, Crook JE, Lesser ER, Schaeverbeke J, Hinkle KM, et al: Selective vulnerability of the nucleus basalis of Meynert among neuropathologic subtypes of Alzheimer disease. JAMA Neurol. 2019 Oct; 77(2):225-33.

32 Mattsson N, Ossenkoppele R, Smith R, Strandberg O, Ohlsson T, Jögi J, et al. Greater tau load and reduced cortical thickness in APOE \&4-negative Alzheimer's disease: a cohort study. Alzheimers Res Ther. 2018 Aug; 10(1):77.

33 Riedel BC, Thompson PM, Brinton RD. Age, APOE and sex: triad of risk of Alzheimer's disease. J Steroid Biochem Mol Biol. 2016 Jun; 160:134-47.

34 Jellinger KA. Neuropathological subtypes of Alzheimer's disease. Acta Neuropathol. 2012 Jan;123(1):153-4.

35 Crary JF, Trojanowski JQ, Schneider JA, Abisambra JF, Abner EL, Alafuzoff I, et al. Primary age-related tauopathy (PART): a common pathology associated with human aging. Acta Neuropathol. 2014 Dec;128(6): 755-66.

36 Janocko NJ, Brodersen KA, Soto-Ortolaza AI, Ross OA, Liesinger AM, Duara R, et al. Neuropathologically defined subtypes of $\mathrm{Al}$ zheimer's disease differ significantly from neurofibrillary tangle-predominant dementia. Acta Neuropathol. 2012 Nov;124(5):68192.

37 Besser LM, Kukull WA, Teylan MA, Bigio EH, Cairns NJ, Kofler JK, et al. The revised National Alzheimer's Coordinating Center's Neuropathology form-available data and new analyses. J Neuropathol Exp Neurol. 2018 Aug;77(8):717-26. 
38 Jellinger KA, Attems J. Challenges of multimorbidity of the aging brain: a critical update. J Neural Transm (Vienna). 2015 Apr;122(4): $505-21$.

39 Josephs KA, Murray ME, Tosakulwong N, Whitwell JL, Knopman DS, Machulda MM, et al. Tau aggregation influences cognition and hippocampal atrophy in the absence of beta-amyloid: a clinico-imaging-pathological study of primary age-related tauopathy (PART). Acta Neuropathol. 2017 May;133(5): 705-15.

40 Jellinger KA, Attems J. Neurofibrillary tanglepredominant dementia: comparison with classical Alzheimer disease. Acta Neuropathol. 2007 Feb;113(2):107-17.

41 Jellinger KA. Commentary on the paper "PART, a Distinct Tauopathy, Different from Classical Sporadic Alzheimer Disease". J Clin Cell Immunol. 2016;7(6):1000480.

42 Bancher C, Egensperger R, Kösel S, Jellinger K, Graeber MB. Low prevalence of apolipoprotein E epsilon 4 allele in the neurofibrillary tangle predominant form of senile dementia. Acta Neuropathol. 1997 Nov;94(5):403-9.

43 Shin WS, Di J, Cao Q, Li B, Seidler PM, Murray $\mathrm{KA}$, et al. Amyloid $\beta$-protein oligomers promote the uptake of tau fibril seeds potentiating intracellular tau aggregation. Alzheimers Res Ther. 2019 Oct;11(1):86.

44 Jellinger KA, Attems J. Prevalence of dementia disorders in the oldest-old: an autopsy study. Acta Neuropathol. 2010 Apr;119(4): 421-33.

45 Whitwell JL, Dickson DW, Murray ME, Weigand SD, Tosakulwong N, Senjem ML, et al. Neuroimaging correlates of pathologically defined subtypes of Alzheimer's disease: a case-control study. Lancet Neurol. 2012 Oct; 11(10):868-77.

46 Whitwell JL, Graff-Radford J, Tosakulwong $\mathrm{N}$, Weigand SD, Machulda M, Senjem ML, et al. [18 F]AV-1451 clustering of entorhinal and cortical uptake in Alzheimer's disease. Ann Neurol. 2018 Feb;83(2):248-57.

47 Byun MS, Kim SE, Park J, Yi D, Choe YM, Sohn BK, et al.; Alzheimer's Disease Neuroimaging Initiative. Heterogeneity of regional brain atrophy patterns associated with distinct progression rates in Alzheimer's disease. PLoS One. 2015 Nov;10(11):e0142756.

48 Risacher SL, Anderson WH, Charil A, Castelluccio PF, Shcherbinin S, Saykin AJ, et al.; Alzheimer's Disease Neuroimaging Initiative. Alzheimer disease brain atrophy subtypes are associated with cognition and rate of decline. Neurology. 2017 Nov;89(21):2176-86.

49 Hwang J, Kim CM, Jeon S, Lee JM, Hong YJ, Roh JH, et al.; Alzheimer's Disease Neuroimaging Initiative. Prediction of Alzheimer's disease pathophysiology based on cortical thickness patterns. Alzheimers Dement (Amst). 2015 Dec;2(1):58-67.
50 Oppedal K, Ferreira D, Cavallin L, Lemstra AW, Ten Kate M, Padovani A, et al.; Alzheimer's Disease Neuroimaging Initiative. A signature pattern of cortical atrophy in dementia with Lewy bodies: A study on 333 patients from the European DLB consortium. Alzheimers Dement. 2019 Mar;15(3):400-9.

51 Persson K, Eldholm RS, Barca ML, Cavallin L, Ferreira D, Knapskog AB, et al. MRI-assessed atrophy subtypes in Alzheimer's disease and the cognitive reserve hypothesis. PLoS One. 2017 Oct;12(10):e0186595.

52 Ferreira D, Pereira JB, Volpe G, Westman E. Subtypes of Alzheimer's disease display distinct network abnormalities extending beyond their pattern of brain atrophy. Front Neurol. 2019 May; 10:524.

53 Machado A, Ferreira D, Grothe MJ, Eyjolfsdottir H, Almqvist PM, Cavallin L, et al. The cholinergic system and treatment response in subtypes of Alzheimer's disease. medRxiv. 2020. Doi: 10.1101/2020.01.23.20018507.

54 Dong A, Toledo JB, Honnorat N, Doshi J, Varol E, Sotiras A, et al.; Alzheimer's Disease Neuroimaging Initiative. Heterogeneity of neuroanatomical patterns in prodromal Alzheimer's disease: links to cognition, progression and biomarkers. Brain. 2017 Mar;140(3):735-47.

55 Poulakis K, Pereira JB, Mecocci P, Vellas B, Tsolaki M, Kłoszewska I, et al. Heterogeneous patterns of brain atrophy in Alzheimer's disease. Neurobiol Aging. 2018 May;65:98-108.

56 Noh Y, Jeon S, Lee JM, Seo SW, Kim GH, Cho $\mathrm{H}$, et al. Anatomical heterogeneity of Alzheimer disease: based on cortical thickness on MRIs. Neurology. 2014 Nov;83(21):1936-44.

57 Shiino A, Watanabe T, Maeda K, Kotani E, Akiguchi I, Matsuda M. Four subgroups of Alzheimer's disease based on patterns of atrophy using VBM and a unique pattern for early onset disease. Neuroimage. 2006 Oct;33(1): $17-26$.

58 Planche V, Coupé P, Helmer C, Le Goff M, Amieva H, Tison F, et al. Evolution of brain atrophy subtypes during aging predicts longterm cognitive decline and future Alzheimer's clinical syndrome. Neurobiol Aging. $2019 \mathrm{Jul}$; 79:22-9.

59 Jeon S, Kang JM, Seo S, Jeong HJ, Funck T, Lee SY, et al. Topographical heterogeneity of Alzheimer's disease based on MR imaging, tau PET, and amyloid PET. Front Aging Neurosci. 2019 Aug;11:211.

60 Zhang X, Mormino EC, Sun N, Sperling RA, Sabuncu MR, Yeo BT; Alzheimer's Disease Neuroimaging Initiative. Bayesian model reveals latent atrophy factors with dissociable cognitive trajectories in Alzheimer's disease. Proc Natl Acad Sci USA. 2016 Oct;113(42): E6535-44.

61 Ferreira D, Verhagen C, Hernández-Cabrera JA, Cavallin L, Guo CJ, Ekman U, et al. Distinct subtypes of Alzheimer's disease based on patterns of brain atrophy: longitudinal trajectories and clinical applications. Sci Rep. 2017 Apr;7(1):46263.
62 Ferreira D, Wahlund LO, Westman E. The heterogeneity within Alzheimer's disease. Aging (Albany NY). 2018 Nov; 10(11):3058-60.

63 Ossenkoppele R, Lyoo $\mathrm{CH}$, Sudre $\mathrm{CH}$, van Westen D, Cho H, Ryu YH, et al. Distinct tau PET patterns in atrophy-defined subtypes of Alzheimer's disease. Alzheimers Dement. 2020 Feb; 16(2):335-44.

64 Iaccarino L, Tammewar G, Ayakta N, Baker SL, Bejanin A, Boxer AL, et al. Local and distant relationships between amyloid, tau and neurodegeneration in Alzheimer's Disease. Neuroimage Clin. 2017 Sep;17:452-64.

65 Tetzloff KA, Graff-Radford J, Martin PR, Tosakulwong N, Machulda MM, Duffy JR, et al. Regional distribution, asymmetry, and clinical correlates of tau uptake on [18F]AV-1451 PET in atypical Alzheimer's disease. J Alzheimers Dis. 2018;62(4):1713-24.

66 McAleese KE, Walker L, Graham S, Moya EL, Johnson M, Erskine D, et al. Parietal white matter lesions in Alzheimer's disease are associated with cortical neurodegenerative pathology, but not with small vessel disease. Acta Neuropathol. 2017 Sep;134(3):459-73.

67 McAleese KE, Colloby S, Attems J, Thomas A, Francis PT. Mixed brain pathologies account for most dementia in the Brains for Dementia Research cohort [abstract]. Neuropathol Appl Neurobiol. 2020;46(Suppl 1):24.

68 McAleese KE, Mohi M, Graham S, Baker G, Walker L, DeCarli C, et al. The aetiology of frontal white matter lesions in Alzheimer's disease are associated with both neurodegenerative and ischemic mechanisms [abstract]. Neuropathol Appl Neurobiol. 2020;46(Suppl 1): 15 .

69 Lowe VI, Lundt ES, Albertson SM, Min HK, Fang P, Przybelski SA, et al: Tau-positron emission tomography correlates with neuropathology findings. Alzheimers Dement. 2020 Mar;16(3):561-571.

70 Shima K, Matsunari I, Samuraki M, Chen WP, Yanase D, Noguchi-Shinohara M, et al. Posterior cingulate atrophy and metabolic decline in early stage Alzheimer's disease. Neurobiol Aging. 2012 Sep;33(9):2006-17.

71 Varol E, Sotiras A, Davatzikos C; Alzheimer's Disease Neuroimaging Initiative. HYDRA: revealing heterogeneity of imaging and genetic patterns through a multiple max-margin discriminative analysis framework. Neuroimage. 2017 Jan;145( Pt B):346-64.

72 Lehmann M, Ghosh PM, Madison C, Laforce R Jr, Corbetta-Rastelli C, Weiner MW, et al. Diverging patterns of amyloid deposition and hypometabolism in clinical variants of probable Alzheimer's disease. Brain. 2013 Mar; 136(Pt 3):844-58.

73 Rabinovici GD, Furst AJ, Alkalay A, Racine CA, O’Neil JP, Janabi M, et al. Increased metabolic vulnerability in early-onset Alzheimer's disease is not related to amyloid burden. Brain. 2010 Feb;133(Pt 2):512-28. 
74 Jack CR Jr, Lowe VJ, Weigand SD, Wiste HJ, Senjem ML, Knopman DS, et al.; Alzheimer's Disease Neuroimaging Initiative. Serial PIB and MRI in normal, mild cognitive impairment and Alzheimer's disease: implications for sequence of pathological events in Alzheimer's disease. Brain. 2009 May;132(Pt 5):1355-65.

75 Mitchell TW, Mufson EJ, Schneider JA, Cochran EJ, Nissanov J, Han LY, et al. Parahippocampal tau pathology in healthy aging, mild cognitive impairment, and early $\mathrm{Alz}$ heimer's disease. Ann Neurol. 2002 Feb; 51(2):182-9.

76 Pascoal TA, Mathotaarachchi S, Shin M, Benedet AL, Mohades S, Wang S, et al.; Alzheimer's Disease Neuroimaging Initiative. Synergistic interaction between amyloid and tau predicts the progression to dementia. Alzheimers Dement. 2017 Jun;13(6):644-53.

77 Giovacchini G, Giovannini E, Borsò E, Lazzeri $\mathrm{P}$, Riondato $\mathrm{M}$, Leoncini $\mathrm{R}$, et al. The brain cognitive reserve hypothesis: A review with emphasis on the contribution of nuclear medicine neuroimaging techniques. J Cell Physiol. 2019 Feb;234(9):14865-72.

78 Stern Y. Cognitive reserve in ageing and Alzheimer's disease. Lancet Neurol. 2012 Nov; 11(11):1006-12.

79 Stern Y, Arenaza-Urquijo EM, Bartrés-Faz D, Belleville S, Cantilon M, Chetelat G, et al.; Reserve, Resilience and Protective Factors PIA Empirical Definitions and Conceptual Frameworks Workgroup. Whitepaper: defining and investigating cognitive reserve, brain reserve, and brain maintenance. Alzheimers Dement. Forthcoming 2018 Sep;S1552-5260 (18)33491-5.

$80 \mathrm{Na}$ HK, Kang DR, Kim S, Seo SW, Heilman KM, Noh Y, et al. Malignant progression in parietal-dominant atrophy subtype of $\mathrm{Alz}$ heimer's disease occurs independent of onset age. Neurobiol Aging. 2016 Nov;47:149-56.

81 Arenaza-Urquijo EM, Vemuri P. Resistance vs resilience to Alzheimer disease: clarifying terminology for preclinical studies. Neurology. 2018 Apr;90(15):695-703.

82 Sui X, Rajapakse JC; Alzheimer's Disease Neuroimaging Initiative. Profiling heterogeneity of Alzheimer's disease using white-matter impairment factors. Neuroimage Clin. 2018;20:1222-32.

83 Nelson PT, Kukull WA, Frosch MP. Thinking outside the box: alzheimer-type neuropathology that does not map directly onto current consensus recommendations. J Neuropathol Exp Neurol. 2010 May;69(5):449-54.

84 Weintraub S, Teylan M, Rader B, Chan KC, Bollenbeck M, Kukull WA, et al. APOE is a correlate of phenotypic heterogeneity in Alzheimer disease in a national cohort. Neurology. 2020 Feb;94(6):e607-12.

85 Galton CJ, Patterson K, Xuereb JH, Hodges JR. Atypical and typical presentations of Alzheimer's disease: a clinical, neuropsychologi$\mathrm{cal}$, neuroimaging and pathological study of 13 cases. Brain. 2000 Mar;123(Pt 3):484-98.
86 Lam B, Masellis M, Freedman M, Stuss DT, Black SE. Clinical, imaging, and pathological heterogeneity of the Alzheimer's disease syndrome. Alzheimers Res Ther. 2013 Jan; 5(1):1.

87 Gorno-Tempini ML, Brambati SM, Ginex V, Ogar J, Dronkers NF, Marcone A, et al. The logopenic/phonological variant of primary progressive aphasia. Neurology. 2008 Oct; 71(16):1227-34.

88 Lee SE, Rabinovici GD, Mayo MC, Wilson SM, Seeley WW, DeArmond SJ, et al. Clinicopathological correlations in corticobasal degeneration. Ann Neurol. 2011 Aug;70(2): $327-40$.

89 McMillan CT, Boyd C, Gross RG, Weinstein J, Firn K, Toledo JB, et al. Multimodal imaging evidence of pathology-mediated disease distribution in corticobasal syndrome. Neurology. 2016 Sep;87(12):1227-34.

90 Di Stefano F, Kas A, Habert MO, Decazes P, Lamari F, Lista S, et al. The phenotypical core of Alzheimer's disease-related and nonrelated variants of the corticobasal syndrome: A systematic clinical, neuropsychological, imaging, and biomarker study. Alzheimers Dement. 2016 Jul;12(7):786-95.

91 van der Flier WM, Pijnenburg YA, Fox NC, Scheltens P. Early-onset versus late-onset Alzheimer's disease: the case of the missing APOE $\varepsilon 4$ allele. Lancet Neurol. 2011 Mar; 10(3):280-8.

92 Petersen C, Nolan AL, de Paula França Resende E, Miller Z, Ehrenberg AJ, Gorno-Tempini ML, et al. Alzheimer's disease clinical variants show distinct regional patterns of neurofibrillary tangle accumulation. Acta Neuropathol. 2019 Oct;138(4):597-612.

93 Ohm DT, Fought AJ, Rademaker A, Kim G, Sridhar J, Coventry C, et al: Neuropathologic basis of in vivo cortical atrophy in the aphasic variant of Alzheimer's disease. Brain Pathol. 2020 Mar;30(2):332-344

94 Josephs KA, Whitwell JL, Tosakulwong N, Weigand SD, Murray ME, Liesinger AM, et al. TAR DNA-binding protein 43 and pathological subtype of Alzheimer's disease impact clinical features. Ann Neurol. 2015 Nov; 78(5):697-709.

95 Galili T, Mitelpunkt A, Shachar N, MarcusKalish M, Benjamini Y. Categorize, cluster, and classify: a 3-C strategy for scientific discovery in the medical informatics platform of the Human Brain Project. In: Džeroski S, Panov P, Kocev D, Todorovski L, editors. Discovery Science. DS 2014. Lecture Notes in Computer Science. Volume 8777. Cham: Springer; 2014.

96 Mitelpunkt A, Galili T, Kozlovski T, Bregman N, Shachar N, Markus-Kalish M, et al. Novel Alzheimer's disease subtypes identified using a data and knowledge driven strategy. Sci Rep. 2020 Jan;10(1):1327.
97 Mukherjee S, Mez J, Trittschuh EH, Saykin AJ, Gibbons LE, Fardo DW, et al.; EPAD Study Group; Investigators from ACT; Investigators from ROS; Investigators from MAP; Investigators from ADNI; Investigators from the University of Pittsburgh ADRC. Genetic data and cognitively defined late-onset Alzheimer's disease subgroups. Mol Psychiatry. 2018 Dec. Doi: 10.1038/ s41380-018-0298-8.

98 Jellinger KA. The enigma of mixed dementia. Alzheimers Dement. 2007 Jan;3(1):4053.

99 Rahimi J, Kovacs GG. Prevalence of mixed pathologies in the aging brain. Alzheimers Res Ther. 2014 Nov;6(9):82.

100 Kapasi A, DeCarli C, Schneider JA. Impact of multiple pathologies on the threshold for clinically overt dementia. Acta Neuropathol. 2017 Aug;134(2):171-86.

101 Boyle PA, Yu L, Wilson RS, Leurgans SE, Schneider JA, Bennett DA. Person-specific contribution of neuropathologies to cognitive loss in old age. Ann Neurol. 2018 Jan; 83(1):74-83.

102 Schneider JA, Arvanitakis Z, Bang W, Bennett DA. Mixed brain pathologies account for most dementia cases in communitydwelling older persons. Neurology. 2007 Dec;69(24):2197-204.

103 Jellinger KA. Clinicopathological analysis of dementia disorders in the elderly-an update. J Alzheimers Dis. 2006;9(3 Suppl):6170.

104 Wang BW, Lu E, Mackenzie IR, Assaly M, Jacova C, Lee PE, et al. Multiple pathologies are common in Alzheimer patients in clinical trials. Can J Neurol Sci. 2012 Sep;39(5): 592-9.

105 Ferreira D, Shams S, Cavallin L, Viitanen M, Martola J, Granberg T, et al. The contribution of small vessel disease to subtypes of Alzheimer's disease: a study on cerebrospinal fluid and imaging biomarkers. Neurobiol Aging. 2018 Oct;70:18-29.

106 DeTure MA, Dickson DW. The neuropathological diagnosis of Alzheimer's disease. Mol Neurodegener. 2019 Aug;14(1): 32.

107 Nelson PT, Dickson DW, Trojanowski JQ Jack CR, Boyle PA, Arfanakis K, et al. Limbic-predominant age-related TDP-43 encephalopathy (LATE): consensus working group report. Brain. 2019 Jun;142(6):150327.

108 McAleese KE, Walker L, Erskine D, Attems J. The impact of concomitant LATE-NC on hyperphosphorylated-s pathology and cognitive decline in Alzheimer's disease [abstract]. Neuropathol Appl Neurobiol. 2020; 46(Suppl 1):37.

109 Gauthreaux K, Bonnett TA, Besser LM, Brenowitz WD, Teylan M, Mock C, et al. Concordance of Clinical Alzheimer Diagnosis and Neuropathological Features at Autopsy. J Neuropathol Exp Neurol. 2020 May;79(5): 465-73. 
110 Plassman BL, Khachaturian AS, Townsend JJ, Ball MJ, Steffens DC, Leslie CE, et al. Comparison of clinical and neuropathologic diagnoses of Alzheimer's disease in 3 epidemiologic samples. Alzheimers Dement. 2006 Jan;2(1):2-11.

111 Cure S, Abrams K, Belger M, Dell'agnello G, Happich M. Systematic literature review and meta-analysis of diagnostic test accuracy in Alzheimer's disease and other dementia using autopsy as standard of truth. J Alzheimers Dis. 2014;42(1):169-82.
112 Fleisher AS, Pontecorvo MJ, Devous MD Sr, Lu M, Arora AK, Truocchio SP, et al.; A16 Study Investigators. Positron emission tomography imaging with [18f]flortaucipir and postmortem assessment of Alzheimer disease neuropathologic changes. JAMA Neurol. 2020 Apr 27;e200528.

113 White LR, Edland SD, Hemmy LS, Montine KS, Zarow C, Sonnen JA, et al. Neuropathologic comorbidity and cognitive impairment in the Nun and Honolulu-Asia Aging Studies. Neurology. 2016 Mar;86(11):1000-8.

114 Jack CR Jr, Bennett DA, Blennow K, Carrillo MC, Dunn B, Haeberlein SB, et al.; Contributors. NIA-AA Research Framework: toward a biological definition of Alzheimer's disease. Alzheimers Dement. 2018 Apr;14 (4):535-62.
115 Nelson PT, Trojanowski JQ, Abner EL, AlJanabi OM, Jicha GA, Schmitt FA, et al "New Old Pathologies": AD, PART, and cerebral age-related TDP-43 with sclerosis (CARTS). J Neuropathol Exp Neurol. 2016 Jun;75(6):482-98.

116 Yokoyama JS, Sirkis DW, Miller BL. C9ORF72 hexanucleotide repeats in behavioral and motor neuron disease: clinical heterogeneity and pathological diversity. Am J Neurodegener Dis. 2014 Mar;3(1):1-18.

117 Respondek G, Grimm MJ, Piot I, Arzberger T, Compta Y, Englund E, et al. Validation of the movement disorder society criteria for the diagnosis of 4-repeat tauopathies. Mov Disord. 2020 Jan;35(1):171-6. 